Herausgeber:

Bundesanstalt für Materialforschung und -prüfung (BAM)

Deutsche Gesellschaft für Zerstörungsfreie

Prüfung (DGZFP)

Deutscher Verband für Materialforschung und -prüfung (DVM)

VDI-Gesellschaft Werkstofftechnik (VDI-W)

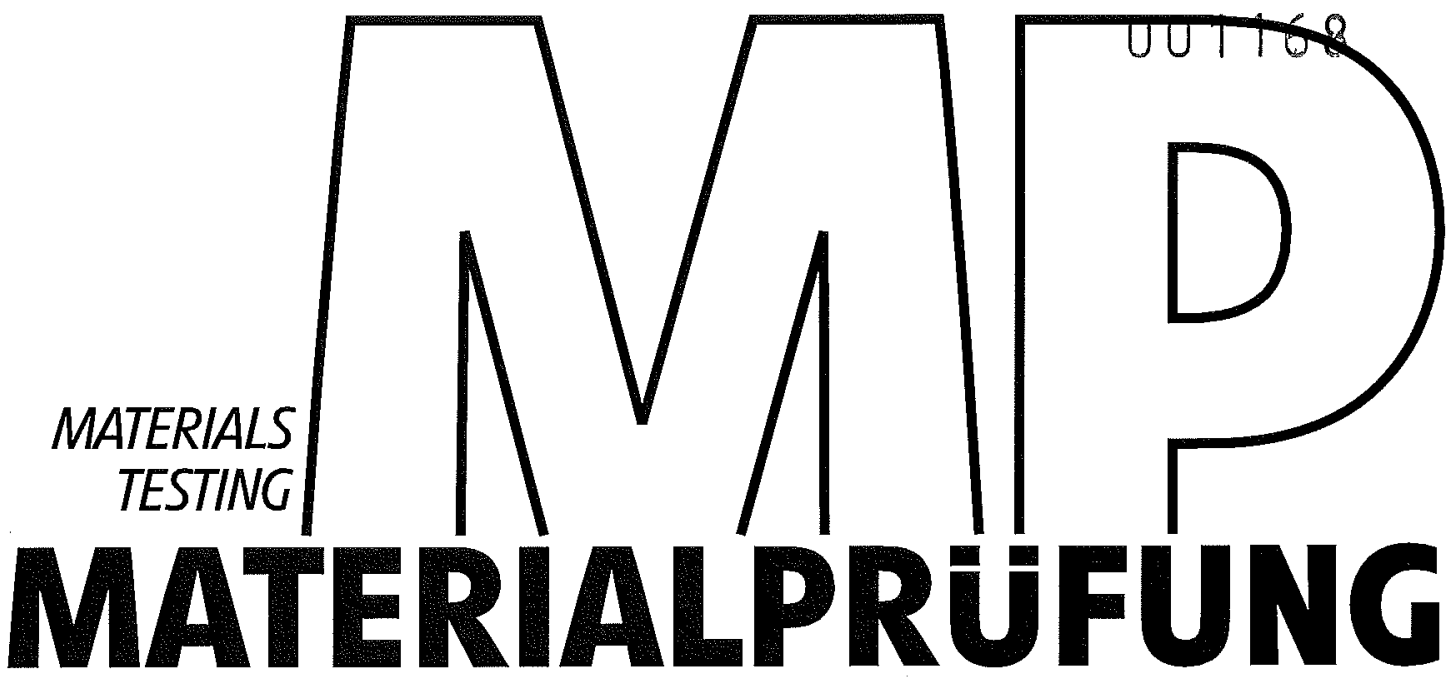

Organ der Bundesanstalt für Materialforschung und -prüfung, Berlin (BAM) Werkstoffe und Bauteile Forschung Prüfung Anwendung

Alle Rechte, auch die des Nachdrucks, der photomechanischen Wiedergabe dieses Sonderdrucks und der Übersetzung, behält sich der Verlag vor.

\title{
Autoren-Fortdruck
}




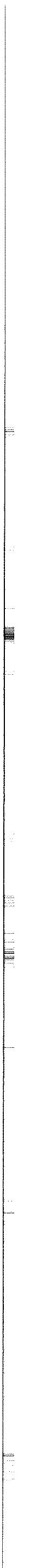




\section{Schubmodul von Kunststoffen bestimmen}

\section{Die Methode der antiklastischen Plattenbiegung}

\author{
Mehdi Farshad und Peter Flüeler, \\ Dübendorf
}

\begin{abstract}
In diesem Beitrag wird über Untersuchungen zur Bestimmung des Schubmoduls von Kunststoffen und Verbundwerkstoffen mit Hilfe der antiklastischen Plattenbiegung (AKPB) berichtet. Die Ergebnisse demonstrieren, daß die Methode der AKPB für die Bestimmung des Schubmoduls von Kunststoffen und Verbundwerkstoffen als geeignet betrachtet werden kann.
\end{abstract}

\section{Der Schubmodul von Kunststoffen}

Die Schubeigenschaften, das heißt der Schubmodul und die Schubfestigkeit sind Werkstoffkennwerte, die für die Charakterisierung und Dimensionierung von Bauelementen notwendig sind. Von Kunststoffen und Verbundwerkstoffen werden dazu neben den Kurzzeit- auch Langzeiteigenschaften und temperaturabhängige Materialkennwerte benötigt.

Bei der Bestimmung mechanischer Kennwerte von thermoplastischen Kunststoffen, Hartschaumstoffen und Verbundwerkstoffen sind generell die materialspezifischen Besonderheiten dieser Werkstoffe, zum Beispiel die möglichen Materialänderungen bei der Probenvorbereitung beziehungsweise die Zeit- und Temperaturabhängigkeit der Materialeigenschaften zu beachten.

Die Kurzzeit- und die temperaturabhängigen Schubmoduln können mit mehreren normierten Prüfmethoden ermittelt werden. Eine gängige Methode ist beispielswiese der Torsionsschwingversuch, bei dem üblicherweise kleine und dünne Proben benutzt werden.

Der sogenannte „Three-Rail-ShearTest" und der Kurzbalkenbiegeversuch sind ebenfalls Methoden zur Schubmodulbestimmung. Diese erfordern jedoch relativ aufwendige Probenvorbereitungen und weisen einige Nachteile bezüglich der Spannungsverteilung auf. Zudem fehlen Methoden zur direkten Bestimmung der Langzeit-Schubmoduln von
Kunststoffen und Verbundwerkstoffen. Eine Besonderheit von Kunststoffen und Verbundwerkstoffen ist ihr zeitund temperaturabhängiges Verhalten. Aus diesem Grund ist die Bestimmung ihrer Materialkennwerte bei unterschiedlichen Temperaturen als Funktion der Zeit unabdingbar. Bekanntlich nimmt der Schubmodul von Kunststoffen mit zunehmender Temperatur ab. Diese Abnahme ist bei der Glasübergangstemperatur $\mathrm{T}_{\mathrm{g}}$ besonders deutlich.

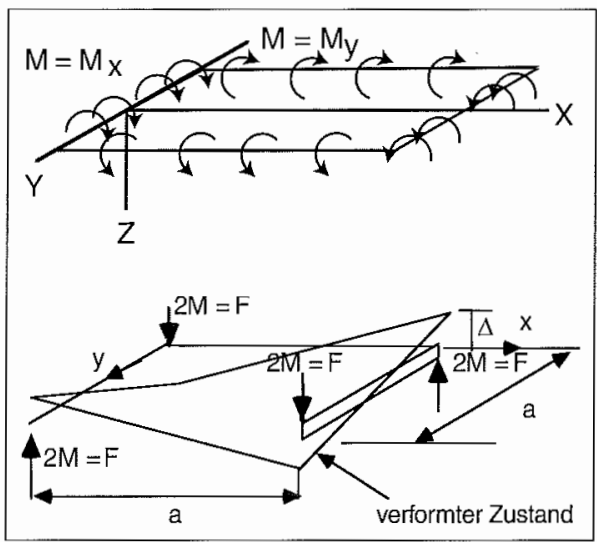

Bild 1. Beispiele von Randkräften, die eine antiklastische Deformation in einer quadratischen Platte erzeugen können, oben: verteilte Biegemomente, unten: Eckkräfte $F$, die statisch äquivalent zu den verteilten Biegemomenten $M$ sind

Zur Bestimmung der Schubeigenschaften von Kunststoffen und Verbundwerkstoffen wurde von der antiklastischen Plattenbiegung eine weiterentwickelte Methodologie abgeleitet. Das grundlegende Prinzip, das für den Bau der AKPB-Versuchseinrichtung verwendet worden ist, basiert auf den Erkenntnissen über das antiklastische Verhalten einer quadratischen Platte.

Gemäß der Plattentheorie deformiert eine quadratische Platte unter torsionserzeugenden Randkräften in einer antiklastischen Verschiebung; das heißt einer Deformation, bei der sich die Platte in zwei unterschiedliche Richtungen verformt. Damit wird eine antiklastische Deformation durch ein reines Schubfeld in Richtung der Plattenkanten erzeugt.

Benutzt man diese Erkenntnisse, kann der Schubmodul der Proben mit Hilfe einer geeigneten Einrichtung, die eine antiklastische Deformation hervorruft, und eines gültigen Modells bestimmt werden. Diese Methode wurde früher zur Bestimmung des KurzzeitSchubmoduls von Holz verwendet [1, 2]. Kürzlich wurde auf der Basis der AKPB ein ISO-Entwurf (Committee Draft) zur Bestimmung des Schubmoduls von Verbundwerkstoffen vorbereitet [3].

Dieser Beitrag faßt die Ergebnisse einer Reihe von Forschungsarbeiten [4 bis 8] mit Beschränkung auf langzeitige und temperaturabhängige Prüfungen von Kunststoffen sowie Verbundwerkstoffen und deren Schubmoduln zusammen.

\section{Antiklastische Plattenbiegung (AKPB)}

Die antiklastische Biegebeanspruchung (Bild 1) bewirkt in einer quadratischen 


\section{Tagungen/Seminare}

6. Tagung „Gefüge und Bruch"

17.3. - 19.3.1999, Bochum Institut für Werkstoffe Werkstoffprüfung, Ruhr Universität Bochum, Tel.: 0234/700-5921, Fax: 0234/7004-400.

- Akkreditierung und Qualitätssicherung von Prüfund Forschungslaboratorien sowie Meß- und Inspektionsstellen 18.3. - 19.3.1999, Eggenstein-Leopoldshafen. FTU Forschungszentrum Karlsruhe GmbH Tel.: 07247/824801, Fax: 07247/824857

Systematische Beurteilung technischer Schadensfälle

22.3. - 26.3.1999, Ermatingen a. Bodensee, Schweiz.

क. DGM ${ }^{4}$

Metallographische Untersuchungsmethoden, Teil B

21.4. - 23.4.1999

Ostfildern.

Technische Akademie

Esslingen,

Tel.: 0711/34008-23

Fax: 0711/34008-43

Werkstoffprüfung und Schadensanalyse an Kunststoffen

27.4. - 28.4.1999

Lüdenscheid.

Kunststoff-Institut

Lüdenscheid,

Tel.: 02351/1064191,

Fax: 02351/1064190.

- 14. Aachener

Stahlkolloquium

29.4. - 30.4.1999, Aachen

Institut für Eisenhütten-

kunde (IEHK) der RWTH

Aachen,

Tel.: 0241/80-5783

Fax: 0241/8888-224.

Angewandte Oberflächen-, Grenzflächenund Düinnschichtanaly-
tik-Methoden und Einsatzbereiche

3.5. - 5.5.1999, München.

V.: VDI ${ }^{2}$

- DVM-Tag „Bauteil '99 Werkstoffe und Verfahren im Wettbewerb“ 5.5. - 7.5.1999, Berlin.

(ब) DVM $^{1}$

Jahrestagung der DGZfP 1999

10.5. - 12.5.1999, Celle.

$\therefore \mathrm{DGZfP}^{4}$

- Deformation und Bruchverhalten von Kunststoffen

23.6. - 25.6.1999,

Merseburg.

Martin-Luther-Universität, Halle-Wittenberg,

Tel.: 03461/46-2777,

Fax: 03461/46-2592.

Einführung in die

Mechanische Werkstoffprüfung

15.9. - 17.9.1999, Osnabrück.

$\because \mathrm{DGM}^{4}$

- Ermüdungsverhalten metallischer Werkstoffe 20.9. - 22.9.1999, Siegen.

DGM ${ }^{4}$

Entstehung, Ermittlung und Bewertung von Eigenspannungen 20.9. - 22.9.1999 Karlsruhe.

(ख) DGM $^{4}$

Messen/ Ausstellungen

13. Control 1999 4.5. - 7.5.1999, Sinsheim. P. E. Schall GmbH, Frickenhausen Tel.: 07025/9206-0, Fax: 07025/9206-620.

- Sensor 99

18.5. - 20.5.1999,

Nürnberg.

ACS Organisations $\mathrm{GmbH}$

Wunstorf,

Tel.: 05033/2015

Fax: 05033/1056

1 DVM Deutscher Verband für Materialforschung und -prüfung, Berlin, Tel.: 030/811-3066, Fax: 030/811-9359.

2 VDI-Bildungswerk GmbH, Düsseldorf, Tel.: 0211/6214-201, Fax: 0211/6214-154.

3 DECHEMA e.V., Frankfurt a.M., Tel.: 069/7564-152, Fax: 069/7564-304.

4 DGM Deutsche Gesellschaft für Materialkunde e.V., Frankfiurt Tel.: 069/7917-755, Fax: 069/7917-733.

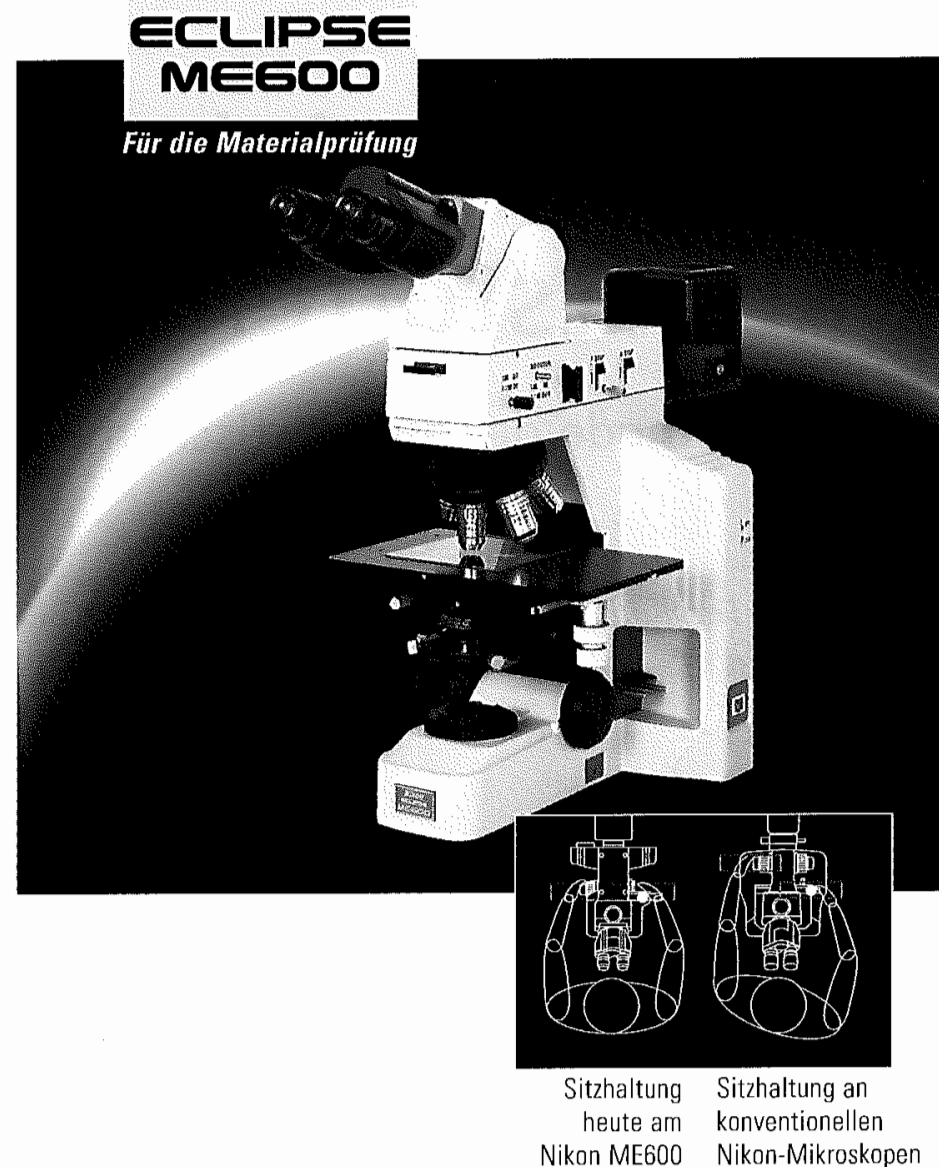

Kompakte Leistung mit Ergonomie

Für Nikon-typische, optische Spitzenleistung der neuen Mikroskope ECLIPSE ME600 für Materialprüfung und -wissenschaft steht die große Vielzahl an „CF“-Infinity-Objektiven für Hellfeld, Dunkelfeld, DIC, Polarisation und Fluoreszenz. Neben der reinen Auflichtversion ME600P können Sie mit dem ME600D auch alle Durchlichttechniken an transparenten Objekten anwenden.

\section{Ergonomisch alles im Blick und im Griff}

Mit Schwenktubus und Höhenanpassung stellen Sie sich die Okulare auf Ihre individuell bequemste Position. Fokus- und Tischbedienung liegen funktional auf einer Höhe zusammen, so daß Sie die Schultern nicht verdrehen müssen. Blendenumschaltungen bei verfahrenswechsel gehen automatisch.

\section{Quantitative Techniken}

Sie können mit dem ME600 Polarisation und Interferometrie durchführen: Für quantitative Charekterisierung Ihrer Prüfstücke und Werkstoffe.

\section{Sinnvolles Zuhehör}

Das Nikon-Angebot für digitale Bildarchivierung und -verarbeitung rundet maßgeschneiderte Problemlösungen für thre Anwendungen genauso ab wie Mehrfach-TV-Module, „FX“.

Photomikrographie-Systeme, Diskussionseinrichtungen und diverse Meßokulare.

Interessiert? Dann fordern Sie bitte ausführliche Unterlagen oder gleich eine Gerätedemonstration an.

Lösungen mit Auflösung

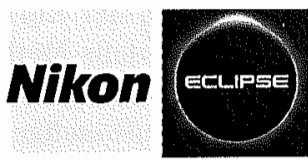

Nikon GmbH, Vertrieb Mikroskope, Instrumente Tiefenbroichen Weg 25, 40472 Düsseldorf Tel : 0211-94-14-0, Fax: 0211-94-14-322 Internet: http://www.nikon.de 
Platte ein zu Stäben analoges Torsionsverhalten. Der Spannungszustand im Querschnitt eines Stabs unter Torsionsbeanspruchung in Stabrichtung entspricht hauptsächlich einem reinen Schubfeld. Um dieses physikalische Phänomen verifizieren zu können, wurde eine Reihe numerischer Finite-ElementSimulationen (FE-Simulationen) der AKPB durchgeführt. In der FE-Analyse wurde das Verhalten einer quadratischen Platte aus PVC-U unter ähnlichen Rand bedingungen wie in Bild 1 unten rechnerisch untersucht. Die Annahmen für die FE-Berechnungen lauten wie folgt: Geometrie: Kantenlänge a $=110 \mathrm{~mm}$; Plattendicke $\mathrm{h}=5 \mathrm{~mm}$, Material: PVC-U, das heißt $\mathrm{E}=3200 \mathrm{MPa}, v=0.35 \rightarrow \mathrm{G}=1185$ $\mathrm{MPa}$, Eckkraft: $\mathrm{F}=30 \mathrm{~N}$ (totale Kraft $2 \mathrm{~F}$ ), Randbedingungen: gelenkig gelagert, Analysetyp: linear elastisch und statisch.

Die FE-Simulation der PVC-Platte zeigt, daß die Schubspannung in der Platten-
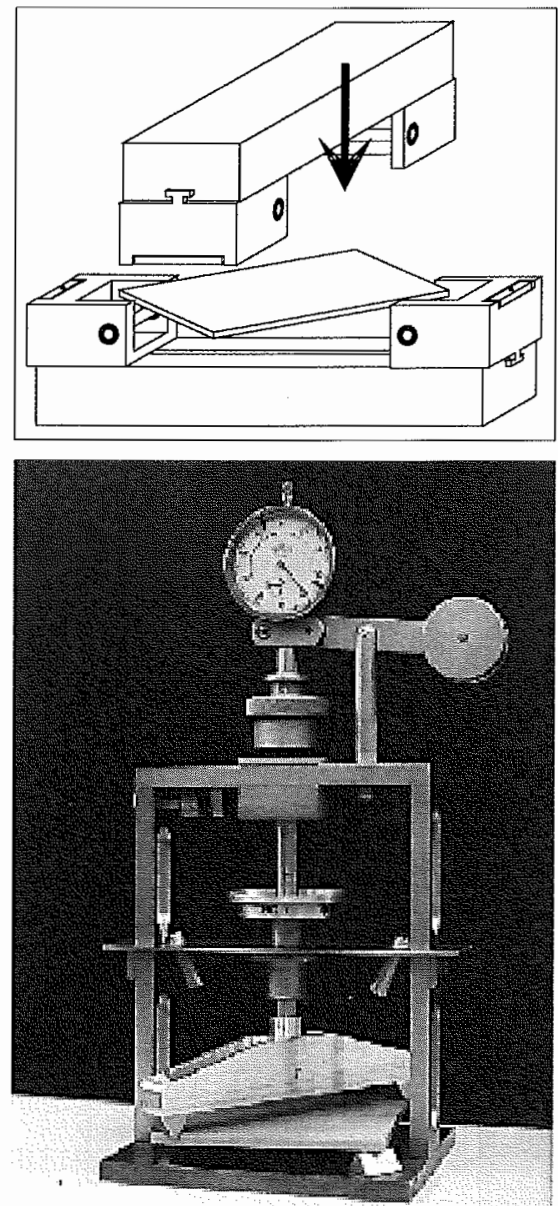

Bild 2. (oben) Konzeptskizze des AKPB-Geräts beziehungsweise der AKPB-Vorrichtung mit variabler Stützweite und beweglichen Auflagern. (Unten) Fotografie des AKPB-Geräts für Langzeitversuche mit maximaler Seitenlänge der Platten von $12 \mathrm{~cm}$

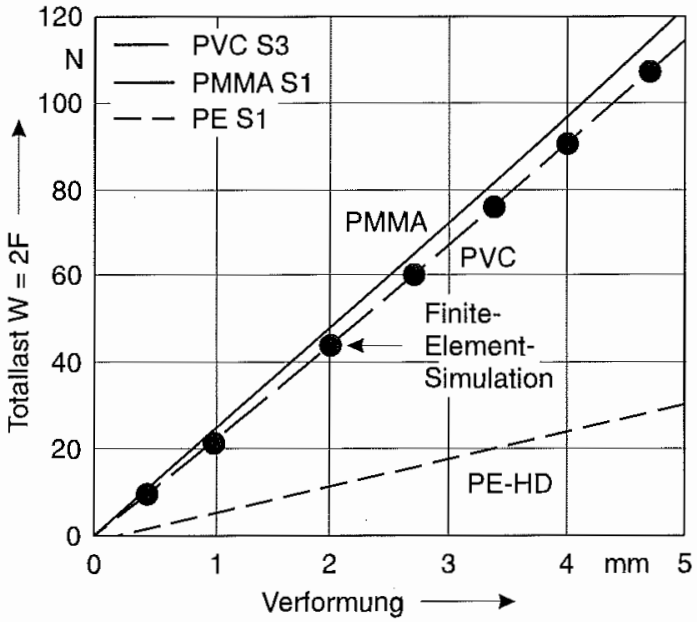

Bild 3. Last-Weg-Diagramme für PE-HD-, PVC- und PMMA-Platten (Thermoplaste) mit den Abmessungen $110 \mathrm{~mm}$ mal $110 \mathrm{~mm} \mathrm{mal}$ $5 \mathrm{~mm}$ mit einigen Datenpunkten der FE-Simulation für PVC; $S 1$ beziehungsweise S3 bezeichnen die Probennuminer ebene weitgehend konstant ist und der Einfluß der Krafteinleitung sich hauptsächlich auf die Ecken beschränkt. Diese Aussage bestätigt, daß die konzipierte AKPB-Versuchsmethodologie ein geeignetes Verfahren zur Bestimmung von Schubeigenschaften sein könnte. Die numerisch berechnete, vertikale Verschiebung an der Plattenecke beträgt 2,8 mm.

\section{AKPB-Versuchseinrichtungen}

Basierend auf dem Prinzip der AKPB wurden zwei Varianten von Prüfeinrichtungen zur Untersuchung von Schubeigenschaften entwickelt. Die eine Variante der AKPB-Vorrichtung dient der experimentellen Untersuchung der Schubeigenschaften auf Universalprüfmaschinen. Die andere Variante der AKPB-Vorrichtung stellt ein unabhängiges Gerät dar, das für Langzeitversuche beziehungsweise Temperatur- und Kriechversuche geeignet ist. Dieses Prüfgerät für Langzeituntersuchungen hat Abmessungen von etwa $30 \mathrm{~cm}$ mal $30 \mathrm{~cm}$ mal $40 \mathrm{~cm}$ und erlaubt die Prüfung von Platten mit einer Seitenlänge bis zu $12 \mathrm{~cm}$.

Bild 2 oben zeigt die Konzeptskizze der AKPB-Versuchsvorrichtung und des AKPB-Geräts. Gemäß diesem Konzept wird die Probe an zwei diagonalen Ecken gelenkig gestützt und an den anderen beiden diagonalen Ecken mit gleichen Eckkräften belastet. Beim AKPB-Gerät in Bild 2 unten erfolgt die variable Belastung der Probe mit Gewichten. Einzelheiten der Entwicklungsphase und der Vorversuche sind dokumentiert [6].

Das AKPB-Gerät zeichnet sich durch geringe Abmessungen sowie einfache Konstruktion und Handhabung aus. Das mobile Gerät kann gut in einer Klima- kammer eingesetzt werden. Mit vernünftigem Aufwand können mehrere solcher Geräte hergestellt und für Langzeitversuche benutzt werden.

Die AKPB-Vorrichtung, die auf einer Universalprüfmaschine installiert werden kann, entspricht der technischen Umsetzung des AKPB-Prinzips in Bild 2 oben. Während einer der zwei senkrecht zueinander angeordneten Ausleger als Auflager dient, wird über den anderen Ausleger die Kraft eingeleitet. Damit können Platten mit Seitenlängen bis zu $30 \mathrm{~cm}$ geprüft werden. Sowohl die Lagerung als auch die Krafteinleitung in die quadratische Probe erfolgen über diagonal angeordnete Walzenlager.

Die durch den diagonalen Ausleger einwirkenden Eckkräfte (Bild 2 oben) können in der AKPB-Vorrichtung durch eine externe Prüfmaschine erzeugt werden. Im Fall einer Universalprüumaschine entsprechen die Eckkräfte dem halben Belastungswert, der von der Kraftmeßdose angezeigt wird.

Beim AKPB-Gerät für Langzeituntersuchungen (Bild 2 unten) werden die Eckkräfte durch Gewichte erzeugt. Die Absenkung der Plattenecken stimmt bei der Universalprüfmaschine mit dem Traversenweg überein. Zusätzlich könnte die Absenkung der Plattenmitte mit Hilfe von Laserstrahlen berührungslos gemessen werden.

\section{Durchgeführte Versuche}

Mit der Methode der AKPB wurden zahlreiche Versuche an unterschiedlichen Kunststoffen und Verbundwerkstoffen durchgeführt. Die Versuche umfaßten Kalibrierung und Ermittlung des Schubmoduls.

Um den Geräteaufbau, die Probengröße und die Laststufen zu optimieren 


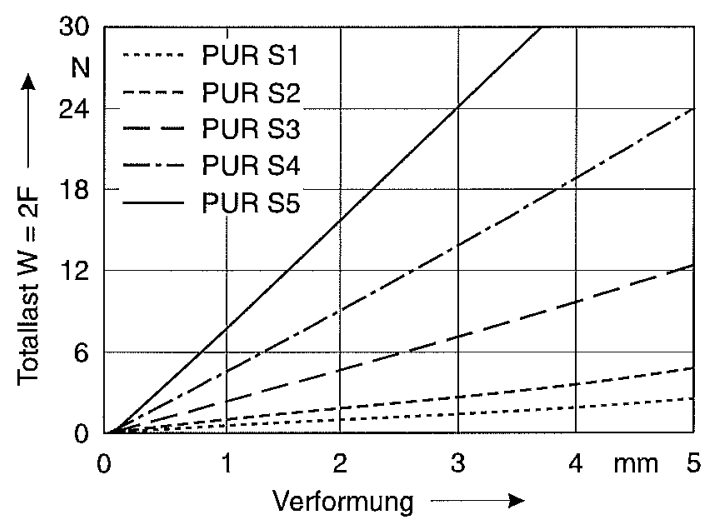

und die Systemfehler zu reduzieren, wurden verschiedene Kalibrierversuche mit Materialien, deren Kennwerte bekannt sind (Stahl, Aluminium und Messing), durchgeführt. Die Ergebnisse dieser Kalibrierversuche wurden mit den Ergebnissen des Torsionsschwing- und des direkten Zugversuchs verglichen. Zum Vergleich mit den FE-Berechnungen wurden auch Versuche an PVC-UPlatten durchgeführt.

Die Experimente zur Ermittlung der Schubeigenschaften von Kunststoffen und Verbundwerkstoffen enthalten die Versuche zur Bestimmung der optimalen Probenabmessungen für jede Klasse von Kunststoffen und Verbundwerkstoffen sowie systematische Experimente zur Bestimmung des Schubmoduls $[4,6]$. In diesem Zusammenhang wurden Polymethylmethacrylat (PMMA), Polycarbonat (PC) und Schaumstoffe, wie Polyvinylchlorid hart (PVC-U), Polyetherimid (PEI), Polymethacrylimid (PMI), Polystyrol (PS-X), und Verbundwerkstoffe wie kohlenstoffaserverstärkte (CFK) und glasfaserverstärkte Kunststoffe (GFK) untersucht.

Um das Potential der AKPB-Methode zur Bestimmung von zeit- und temperaturabhängigen Schubeigenschaften von Kunststoffen zu untersuchen, wurden eine Reihe von AKPB-Versuchen an Kunststoffen bei Temperaturen von $23^{\circ} \mathrm{C}, 40^{\circ} \mathrm{C}, 60^{\circ} \mathrm{C}$ und $80^{\circ} \mathrm{C}$ in einer Klimakammer durchgeführt.

Vergleichende Versuche wurden auch mit der Torsionsschwingmethode ausgeführt. Zur Überprüfung des AKPBGeräts auf seine Eignung für den Langzeitversuch und zur Bestimmung der Zeitabhängigkeit des Schubmoduls wurden AKPB-Kriechversuche an PVC-UPlatten durchgeführt.

Je nach Materialtyp wurden die AKPBExperimente mit unterschiedlichen Last- und Verformungswerten realisiert.
Bild 4. Last-Weg-Diagramme aus AKPBVersuchen für PUR-Hartschaum mit einer Dichte von rund $100 \mathrm{~kg} / \mathrm{m}^{3}$ mit verschiedenen Plattendicken; S1 bis S5 bezeichnen die Probennummer

Die Experimente wurden bis in den Bereich großer Verformungen (nichtlinearer Bereich) weitergeführt. Verschiebungsraten lagen typisch zwischen $1 \mathrm{~mm} /$ min und $5 \mathrm{~mm} / \mathrm{min}$. Für die Schubmodulbestimmung nach der AKPB-Theorie wurde jeweils nur der lineare Bereich dieser Diagramme verwendet.

Die Anforderung, daß die Last-Weg Diagramme eines elastischen Materials einen linearen Teil enthalten sollten, konnte durch geeignete Probenabmessungen (Breite-Dickenverhältnis), die Versuchsvorrichtung und die Belastungs- und Verschiebungswerte realisiert werden.

\section{Experimentelle Daten}

Die Versuchsdaten der AKPB-Methode werden als Last-Weg-Diagramme dargestellt. Die vertikale Achse dieser Diagramme bezeichnet die totale Querkraft und die horizontale Achse stellt die Verschiebung der Plattenecke senkrecht zur Plattenebene dar.

Thermoplastische Kunststoffe Bild 3 zeigt die Ergebnisse der Kurzzeitversuche an quadratischen PVC-U-, PE-HD- und PMMA-Platten. Die Abmessungen der Proben wurden mit $110 \mathrm{~mm}$ mal $110 \mathrm{~mm}$ mal $5 \mathrm{~mm}$ gewählt, die
Stützweite betrug bis zu $100 \mathrm{~mm}$. Die Ergebnisse der FE-Berechnungen für eine PVC-Platte sind ebenfalls in dieses Diagramm eingetragen. Zwischen den experimentellen Ergebnissen und der theoretischen Simulation besteht eine gute Übereinstimmung.

\section{PUR-Hartschaumstoffe}

Einen wesentlichen Vorteil bietet die AKPB-Methode bei der Prüfung von Werkstoffen, bei denen die Probenvorbereitung und Versuchsdurchführung unerwünschte Nebenwirkungen auf das Versuchsergebnis haben können. Schaumstoffe und einige andere Materialien mit Zellenstruktur gehören zu dieser Werkstoffklasse. Bild 4 zeigt die Ergebnisse der AKPB-Versuche an PURHartschaum. Dieses Bild stellt die LastWeg-Diagramme für quadratische PURPlatten mit Flächenabmessungen von $100 \mathrm{~mm}$ mal $100 \mathrm{~mm}$ mit verschiede nen Dicken im Dichtebereich von rund $100 \mathrm{~kg} / \mathrm{m}^{3}$ dar.

\section{Verbundwerkstoffe}

Bild 5 zeigt die Ergebnisse von Kurzzeitversuchen an $0 / 90-$ und $\pm 45^{\circ}$-GFKLaminaten. Die GFK-Probe S1 ist eine 0/90-Probe und S2 eine \pm 45-Probe. In Bild 5 sind auch die Ergebnisse von AKPB-Versuchen an Kartonpapier (HPA) und Papierlaminaten (HGE) enthalten. Weil das Ziel dieser Versuche die Erprobung der Versuchsmethode für andere Materialtypen war, wurde eine detaillierte Charakterisierung dieser Materialien nicht durchgeführt.

\section{Parametrische Modellierung}

Für die Auswertung der experimentellen Daten wurden einfache, parametrische Modelle entwickelt, die auch vorteilhaft für die Optimierung der Ver-

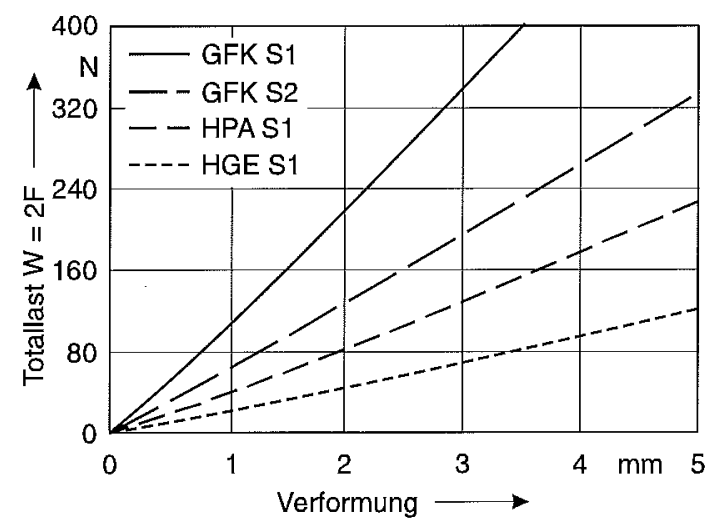

Bild 5. Last-Weg-Diagramme für GFK-Laminate (GFK), Papierlaminat (HGE) und Karton (HPA); $S 1$ beziehungsweise $S 2$ bezeichnen die Probennummer 
suchsparameter verwendet werden können.

Die Methodologie der AKPB entspricht einer quadratischen Platte, die an zwei diagonalen Stützpunkten gelagert ist und bei der an den Ecken der anderen Diagonalen ein Paar vertikaler Kräfte $\mathrm{F}$ einwirkt (Bild 1). Die totale vertikale Kraft beträgt $W=2 \cdot F$. Nach der klassischen Plattentheorie kann die Verschiebung $\Delta$ an der Ecke dieser Platte mit Hilfe der folgenden Formel berechnet werden [6]:

$\Delta=\frac{\mathrm{Fa}^{2}}{4 \mathrm{D}(1-v)}$,

wobei a die Länge und $\mathrm{D}$ die Biegesteifigkeit der Platte bezeichnen. Die Beziehung zwischen D, der Plattendicke $h$ und den Materialeigenschaften lautet:

$\mathrm{D}=\frac{E h^{3}}{12\left(1-v^{2}\right)}$,

wobei E der Elastizitätsmodul und $v$ die Querkontraktionszahl der Platte bedeuten. Durch Umformung der Gleichungen 1 und 2 erhält man:

$v=\frac{\mathrm{Eh}^{3} \Delta}{3 \mathrm{Fa}^{2}}-1$

Für ein isotropes Material gilt $G=\frac{E}{2(1+v)}$.

Daher folgt:

$\mathrm{G}=\frac{3 \mathrm{Fa}^{2}}{2 \Delta \mathrm{h}^{3}}$

Für anisotrope Materialien kann eine ähnliche Gleichung hergeleitet werden. Gleichung 4 basiert auf den Voraussetzungen der klassischen Plattentheorie, bei der die Schubdeformation vernachlässigt wird. In den durchgeführten Versuchen wurde ein Verhältnis der Plattendicke zur Plattenlänge von $1 \mathrm{zu} 20$ gewählt. Bei diesem Verhältnis kann der Einfluß der Schubdeformation vernachlässigt werden.

\section{Ergebnisse der AKPB-Untersuchungen}

\section{Kurzzeit-Schubmodul}

Die mit Hilfe der AKPB-Vorrichtung gewonnenen experimentellen Daten wurden mittels parametrischer Modelle ausgewertet. In Tabelle 1 sind die Ergeb-

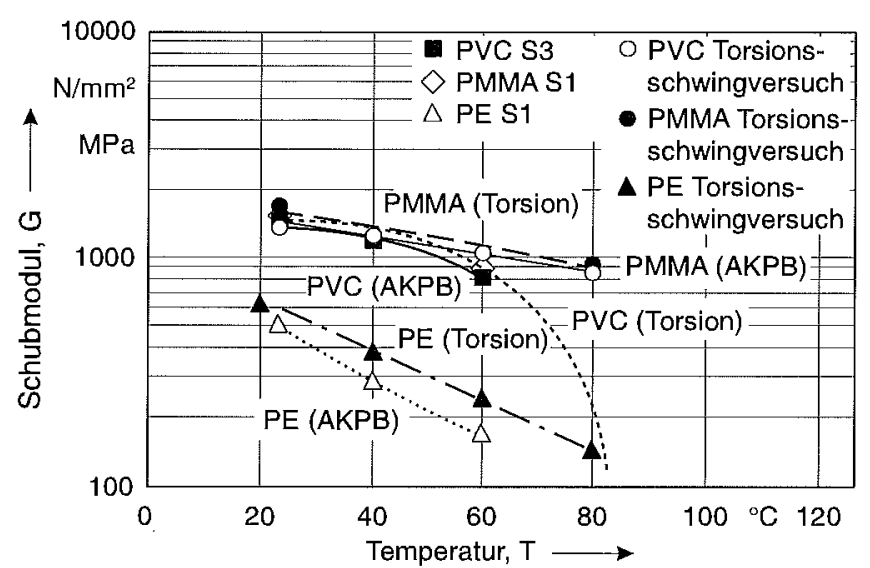

Bild 6. Der Realteil des Schubmoduls einiger Kunststoffe als Funktion der Temperatur nisse der Schubmodul-Bestimmungen zusammengefaßt.

Temperaturabhängiger Schubmodul Mit dem in Bild 2 unten dargestellten AKPB-Gerät für Langzeituntersuchungen wurde der temperaturabhängige Schubmodul einiger Kunststoffe ermittelt. Diese Messungen erfolgten mit dem mobilen AKPB-Gerät in einer Temperaturkammer bei Temperaturen von $+23^{\circ} \mathrm{C},+40^{\circ} \mathrm{C},+60^{\circ} \mathrm{C}$ und $+80^{\circ} \mathrm{C}$. Die ermittelten Schubmodule sind in Bild 6 als Funktion der Temperatur aufgetragen. Als Vergleich zu den AKPB Messungen ist in diesem Diagramm der Schubmodul aus Torsionsschwingversu- chen an den gleichen Kunststoffen aufgeführt.

Das in Bild 2 unten abgebildete AKPBGerät für Langzeituntersuchungen wurde auch zur Bestimmung des Kriechverhaltens von Kunststoffen und des zeitlichen Verlaufs des Schubmoduls eingesetzt. In Bild 7 ist die zeitliche Verformung einer Plattenecke für PVC-UPlatten unter konstanter Eckkraft von 12,67 N wiedergegeben.

Die Auswertung des Diagramms in Bild 7 ergibt mit Hilfe der AKPB-Theorie die Kriechkurve des Schubmoduls. Bild 8 zeigt die Variation des Schubmo-
Zeitabhängiger Schubmodul

\begin{tabular}{|l|c|}
\hline Material & Schubmodul \\
\cline { 2 - 2 } & $\mathrm{MPa}$ \\
\hline Polymethylmethacrylat (PMMA) & 1210,0 \\
\hline Polycarbonat (PC) & 870,0 \\
\hline Polyäthylen hoher Dichte (PE-HD) & 360,0 \\
\hline Unplastifiziertes Polyvinylchlorid (PVC-U) & 1170,0 \\
\hline Polyvinylchlorid; PVC-Hartschaum mit $75 \mathrm{~kg} / \mathrm{m}^{3}$ & 23,7 \\
\hline Polyurethan (PUR) Hartschaum mit $100 \mathrm{~kg} / \mathrm{m}^{3}$ & 33,0 \\
\hline Polyetherimid (PEI) Hartschaum mit $80 \mathrm{~kg} / \mathrm{m}^{3}$ & 22,5 \\
\hline Polyetherimid (PEI) Hartschaum mit $65 \mathrm{~kg} / \mathrm{m}^{3}$ & 15,0 \\
\hline Polyetherimid (PEI) Hartschaum mit $50 \mathrm{~kg} / \mathrm{m}^{3}$ & 12,5 \\
\hline Polymethacrylimid (PMI) Hartschaum mit $60 \mathrm{~kg} / \mathrm{m}^{3}$ & 33,8 \\
\hline Polystyrol (PS-X) Hartschaum mit $40 \mathrm{~kg} / \mathrm{m}^{3}$ & 9,2 \\
\hline Glasfaserverstärktes Polyester (GF-UP) & 5200,0 \\
\hline $\begin{array}{l}\text { Kohlenstofffaserverstärktes Epoxidharz (CF-EP, T700, Toray), } \\
\text { unidirektional }\end{array}$ & 4630,0 \\
\hline $\begin{array}{l}\text { Corian, PMMA gefüllt mit Aluminiumhydroxid, Al }(\mathrm{OH})_{3}, \\
\text { mit1780 kg/m }{ }^{3} \text {, 6 mm dick }\end{array}$ & 3650,0 \\
\hline
\end{tabular}

Tabelle 1. Schubmodulwerte einiger Kunststoffe und Verbundwerkstoffe ermittelt mit der AKPB-Methode 


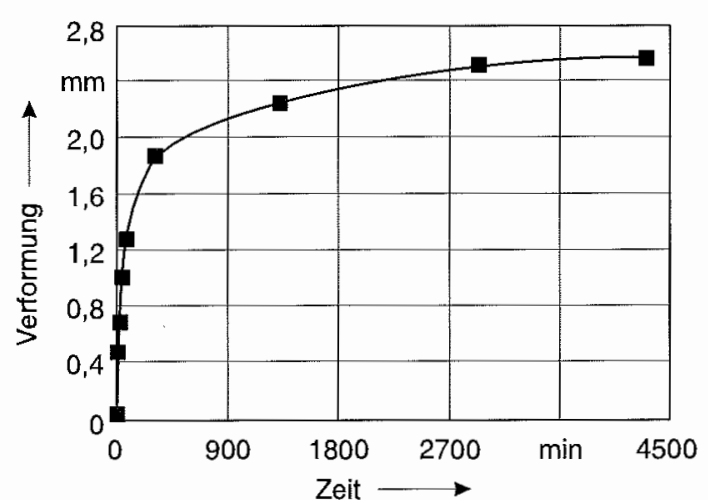

Bild 7. Verformung der Plattenecke als Funktion der Zeit unter konstanter Eckkraft von 12,67 N, die totale Kraft beträgt $25,34 \mathrm{~N}$

duls für PE-HD als Funktion der Zeit. Sie stellt den Kriechmodul des PE-HD-Materials dar.

\section{Diskussion und Schlußfolgerungen}

Obwohl das Prinzip der AKPB bereits seit einiger Zeit bekannt ist, erforderte die Anwendung dieses Prinzips, insbesondere zur Ermittlung des LangzeitSchubmoduls von Kunststoffen, Verbundwerkstoffen und Schaumstoffen, einige Forschungs- und Entwicklungs arbeiten. Sie können als Grundlage zur Einführung einer normierten AKPBPrüfmethode dienen.

Zur Simulation der AKPB wurde eine analytische Beziehung für die benötigten Parameter hergeleitet. Zusätzlich wurden einige Finite-Element-Simulationen der Versuche durchgeführt, die mit den experimentellen Ergebnissen sehr gut übereinstimmen.

Die Methode der AKPB stellt ein einfaches und zuverlässiges Mittel zur Bestimmung der Schubeigenschaften von Kunststoffen und Verbundwerkstoffen dar. Die wesentlichen Vorteile der AKPB-Methode sind: Relativ reines Schubfeld in der Plattenebene infolge der AKPB-Beanspruchung, einfache Versuchseinrichtung, minimaler Aufwand für Probenvorbereitung und damit niedrige Materialbearbeitungskosten, Zeitersparnis bei der Versuchsdurchführung, direkte parametrische Ermittlung des Schubmoduls, geringe Streuung der Meßwerte, Möglichkeit zur Bestimmung der zeit- und temperaturabhängigen Schubeigenschaften von Kunststoffen und Verbundwerkstoffen mit niedrigem Aufwand und Potential zur Bestimmung der Schubeigenschaften von Klebstoffen.

Die AKPB-Methode und deren technische Realisierung im AKPB-Gerät beziehungsweise in der AKPB-Vorrichtung scheinen eine zuverlässige Methode zu liefern, mit der Kurzzeit- und LangzeitSchubmoduln von Kunststoffen und Verbundwerkstoffen bestimmt werden können.

Mit den an der EMPA realisierten, einfach aufgebauten Versuchseinrichtungen können Untersuchungen an Proben mit unterschiedlichen Abmessungen durchgeführt werden. Die Geräte sind dabei besonders zur Schubuntersuchung von Werkstoffen geeignet, bei denen die Probenvorbereitung Änderungen in den Materialeigenschaften bewirken kann. Schaumstoffe sind ein typisches Beispiel der Werkstoffklasse, bei der das AKPB-Gerät für Kurz- und Langzeitprüfungen eingesetzt werden kann. Mit diesem System werden zudem
Bild 8. Variation des Kriechmoduls von PE-HD als Funktion der Zeit, die Kurve stammt aus einer Anpassung an die Meßwerte (Punkte)

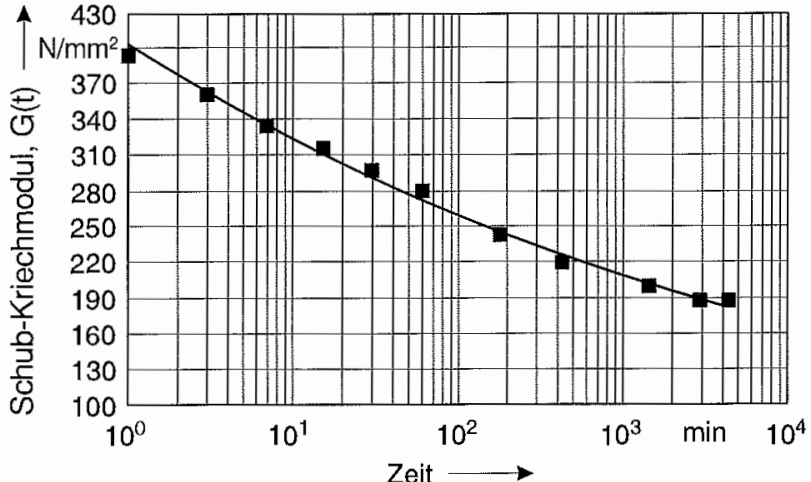

die Kosten der Probenvorbereitung reduziert.

Um die Kosten für Langzeitversuche reduzieren zu können, wurde das in Bild 2 unten dargestellte AKPB-Gerät bewußt als einfache, mobile Konstruktion ausgelegt. Es ist möglich, mehrere solcher Geräte mit relativ kleinem Aufwand herzustellen und in einer Klimakammer zu installieren.

Die AKPB-Vorrichtung zur Installation in Universalprüfmaschinen bietet den Vorteil, daß genaue Untersuchungen an unterschiedlichen Probentypen und Probenabmessungen unter Benutzung der präzisen Last-Weg-Messung einer Universalprüfmaschine durchgeführt werden können. Dies führt zu präziseren Kurzzeitdaten als mit dem AKPBGerät für Langzeituntersuchungen.

Generell liefern beide Vorrichtungen reproduzierbare Ergebnisse, die miteinander sehr gut korrelieren. Sie haben sich somit bei ihrer Erprobung bewährt.

\section{Literatur}

1 Biblis, E. J.; Lee, W. C.: Simplification on the Experimental Method for Determining Plate Shear Modulus of Plywood and Particle Board. Forest Products Journal 26 (1976) 4, S. 38-41

2 ASTM D 3044: Standard Test Method for Shear Modulus of Plywood. American Society for Testing and Materials, Philadelphia, 1994

3 Committee Draft ISO/CD 15310 (ISO/TC 61/SC 13 N 356): Reinforced PlasticsDetermination of In-Plane Shear Modulus by the Plate Twist Method. (Item 681). ISO, Geneve 1997

4 Farshad, M.; Wildenberg, M. W.; Flüeler, P. Determination of Shear Modulus and Poisson's Ratio of Polymers and Foams by Anticlastic Plate Bending Method. Materials and Structures 30 (1997) July, S. 377-382

5 Farshad, M.: Investigation of Adhesives Properties and Mode IIl Crack Growth with Anticlastic Plate Bending Method. In: Pick, R. (Hrsg.): Proceedings of European Conference on Macromolecular Physics Surfaces and interfaces in Polymers and Composites. Volume 21B. European Physical Society, Lausanne 1997, S. 145-146

6 Determination of Shear Modulus and Poisson's Ratio by Anticlastic Plate Bending Method. EMPA-Bericht Nr. 157'112. EMPA, Dübendorf 1994

7 Farshad, M.; Flüeler, P.: Application of the Anticlastic Plate Bending Method for Investigation of the Mode III Fracture Toughness. Engineering Fracture Mechanics 60 (1998) 5-6, S. 597-603

8 Farshad, M.: Design and Analysis of Shell Structures. Kluwer Academic Publishers, Dordrecht 1992, S. 5 


\section{Die Autoren dieses Beitrags}

Professor Dr. Mehdi Farshad, geboren 1942, studierte an der Universität Teheran Bauingenieurwesen mit dem AbschluB des B. S., an der Columbia University mit den Abschlüssen M.S. und C.E. und erwarb an der Stanford University den Ph. D. Beginnend ab 1970 war er als Professor an der Universität Shiraz im Iran und als Gastprofessor an der University of Toronto tätig und an mehreren Forschungsprojekten beteiligt. Seit 1991 arbeitet er an der EMPA im Bereich Kunststoffe und Verbundstrukturen und leitet die Ingenieurgruppe der Abteilung Kunststoffe/Composites. 1996 verlieh ihm die ETH den Rang eines Titularprofessors. Seit 1991 hält er an der ETH Vorlesungen über Stabilität von Schalen und Verbundsystemen, Biomechanik und Finite-Element Simulationen.

Peter Flüeler, geboren 1943, studierte an der ETH Zürich Bauingenieuwesen, diplomierte 1969 und arbeitete zuerst als projektierender Ingenieur im Hoch- und Tiefbau. Beginnend mit dem Jahr 1972 ist er in der EMPA Dübendorf als Prüf- und Forschungsingenieur im Bereich des Massivbaus tätig. Seit 1979 leitet er nach Weiterbildung am MIT im Bereich Kunststoffe/
Composites die Abteilung Kunststoffe/Composites an der EMPA, wo er Fragen der Kunst stoffanwendung, der Bruchmechanik und deren spezielle Prüftechnik bearbeitet. Seit 1986 ist er an der ETH-I Zürich auch nebenamtlicher Dozent für Kunststoffe und deren Anwendungen in der Architektur, im Bauingenieur bereich und in den Werkstoffwissenschaften.

\section{Summary}

Determination of the Shear Modulus of Polymers and Laminated Materials with the Method of Anticlastic Plate Bending. This paper deals with the determination of shear moduli of polymers and composites based on anticlastic plate bending (ACPB). Calibration tests on metallic specimens were used to determine the suitable specimen dimensions. Long-term and temperature-dependent shear moduli of PVC-U, PE-HD, and PMMA were measured. In some cases, the test results from $\mathrm{ACPB}$ were compared with those obtained from torsion pendulum and tensile tests on identical materials. These comparisons yielded good agreement. The results show that the ACPB method can be regarded as a reliable and yet simple test for the determination of the shear moduli of polymers and composites.

\section{Innovation und Förderung}

Die meisten kleinen und mittelständischen Unternehmen finanzieren ihren Innovationsaufwand vorrangig über erwirtschaftete Gewinne. Das geht aus einer Untersuchung des Fraunhofer-Instituts für Systemtechnik und Innovationsforschung, ISI, Karlsruhe, hervor. Öffentliche Fördermittel und Bankkredite spielen dabei eine untergeordnete Rolle. Neben Forschung und Entwicklung beinhaltet Innovationsaufwand auch die Markteinführung und Produktionsvorbereitung.

Die Förderfibel 1998 des Bundesministeriums für Bildung und Forschung nennt Programme, die in kleinen und mittleren Unternehmen Forschung und Entwicklung fördern sollen. Davon eignen sich laut Handelsblatt insbesondere das ERP-Innovationsprogramm und das Beteiligungskapital für kleine Technologieunternehmen (BTU), dazu, Innovationsvorhaben abzusichern.

In der neuen Bundesregierung haben sich die Zuständigkeiten im Bereich der Innovationsförderung verschoben. Aufgaben, die bislang das Bundesministerium für Bildung und Forschung wahrnahm, wurden auf das Bundesministe- rium für Wirtschaft und Technologie übertragen. Dazu gehören unter anderem die indirekte Innovations- und Technologieförderung für kleine und mittelständische Unternehmen, die Existenzförderung für neue Unternehmen und die angewandte Energieforschung, Förderung neuer und erneuerbarer Technologien. Weiterhin vereinbarten beide Minister in allen Bereichen der Forschungs- und Technologieförderung verstärkte Kooperation.

Die EU will mit ihrem fünften Rahmenprogramm die angwandte Forschung stärker berücksichtigen. Mit Aufrufen zur Abgabe von Anträgen, die sich auf das fünfte Programm beziehen, ist im Februar zu rechnen. Um durch ein EU-Programm unterstützt zu werden, muß ein Projekt verschiedene Voraussetzungen erfüllen. Dazu gehört zum Beispiel eine Zusammenarbeit über nationale Grenzen hinaus. Für das Programm „Förderung der Innovation und der Einbeziehung von kleiner und mittelständischer Unternehmen" sollen 363 Milliarden Euro zur Verfügung gestellt werden. Informationen darüber bekommt man zum Beispiel bei den
Euro Inof Centres (EIC), die bei vielen Industrie- und Handelskammern zu finden sind oder auch beim SteinbeisTransferzentrum Technologiebewertung und Innovationsberatung TIB in Mannheim (Telefon 0621/83375-12).

Warum manche Unternehmen mit ihren Innovationen erfolgreich sind, andere wiederum scheitern, haben österreichische Wissenschaftler um Professor Jaberg vom Institut für hydraulische Strömungsmaschinen der TU Graz in Zusammenarbeit mit dem Fraunhofer-Institut für Fabrikbetrieb und -automatisie-rung, IFF, in Magdeburg untersucht. An dem Projekt waren vier mittelständische Unternehmen beteiligt. Dabei haben sich laut Handelsblatt vom 23. 12. 98 einige Voraussetzungen und Handlungsweisen herauskristallisiert, die einen erfolgreichen Innovationsprozeß kennzeichnen. Wichtig sind zum Beispiel offene Unternehmenskultur, lernende Organisationen, Kundennähe, Innovationstreiber auf oberster Firmennähe und objektive Bewertung von Markt und Technologie und der eigenen Position. Die Untersuchung wird weitergeführt und soll noch weitere Unternehmen einbeziehen. 


\title{
Strukturelle Sicherheit quantifizieren
}

\section{Das Damage Tolerance Design Konzept}

\author{
Günter Marci, Köln
}

\section{Das "Safe Life“-Konzept}

Historisch gesehen erfolgte die Absicherung technischer Bauteile gegen Bruch durch empirisch gewonnene Erkenntnisse, die sich bei der Dimensionierung und der Wahl des Werkstoffs für ein betroffenes Bauteil dokumentierten. Der Grundgedanke war, daß die während der vorgesehenen Betriebszeit im Bauteil ablaufenden Schädigungsprozesse nicht zu einem vorzeitigen Versagen führen dürften („Safe Life“-Konzept). Als Schädigungsprozesse, um die wichtigsten zu nennen, müssen berücksichtigt werden: bei höheren Temperaturen: Kriechen, Oxidation, eventuell werkstoffspezifische Änderungen im Werkstoff selbst, zum Beispiel Wasserstoffversprödung, Heißgaskorrosion,

in korrosiver Umgebung: korrosiver Abtrag, Lochfraß, Wasserstoffversprödung, Spannungsrißkorrosion, Schwingungsrißkorrosion,

- unter betrieblichen Belastungen: Rißentstehung, Rißwachstum unter zyklischer und/oder statischer Belastung, Bruch unter maximaien Betriebszuständen oder Störfall-Situationen.

Nach allgemeiner Erfahrung nimmt das Versagen von Bauteilen seinen Ausgangspunkt von Werkstoff- beziehungsweise Fertigungsfehlern. Diese Fehler wachsen unter dem Einfluß der Betriebsbedingungen bis zum Bruch. Somit stellt sich intuitiv die Aufgabe, ein

\begin{abstract}
Das ICE-Unglück in Eschede wirft Fragen nach der strukturellen Sicherheit hoch belasteter Bauteile in neuartigen Verkehrs- und Energiesystemen auf. Dieser Beitrag versucht eine Antwort auf die Frage zu geben, ob das in Deutschland wie auch in Europa vorherrschende strukturelle Sicherheitskonzept, das „Safe Life“-Konzept, den Sicherheitsanforderungen, die wir heute an moderne technische Systeme stellen, noch gerecht werden kann.
\end{abstract}

möglichst fehlerfreies Bauteil herzustellen und die Auswirkung der Betriebsbedingungen auf die Schädigung des Bauteils so einzuschränken, daß ein Bauteilversagen in der vorgesehenen Betriebszeit, der geplanten Lebensdauer des Bauteils, ausgeschlossen ist.

Diese Bemühungen zur Bruchverhinderung bezeichnet man als „Safe Life“Konzept. Obwohl die Herstellung eines fehlerfreien Bauteils aus metallurgischer Sicht unmöglich ist, da Versetzungen, Ausscheidungen und Einschlüsse nicht vermeidbar sind, behandelt man beim „Safe Life"-Konzept Bauteile als quasi fehlerfrei. Begründet wird diese Annahme durch die Hypothese, daß die möglicherweise vorhandenen Bauteilfehler nach Durchlaufen eines kontrollierten und geprüften Herstellungsverfahrens niemals zum Bruch des Bauteils führen dürfen. Vorausgesetzt wird bei dieser Hypothese, daß das Bauteil spannungsmäßig richtig bemessen ist.

Das „fehlerfreie Bauteil" wird also durch Konsens definiert, wobei das „Definieren durch Konsens" von einer Industriesparte zur anderen sehr unterschiedliche Maßnahmen in bezug auf Herstellung, Prüfung und spannungsmäßige Bemessung beinhalten kann.

Da aber generell von einem quasi fehlerfreien Bauteil ausgegangen wird, erübrigt sich die Frage nach einem quantifizierbaren Anfangspunkt der Lebensdauer. Das "Safe Life"-Konzept startet mit einem quasi fehlerfreien Bauteil. Die nutzbare Lebensdauer ergibt sich als Bruchteil der Zeit, die erforderlich wäre, dieses Bauteil unter den Betriebsbelastungen zu zerstören.

Die nutzbare Lebensdauer multipliziert mit dem Sicherheitsfaktor ergibt die theoretische Lebensdauer. Es ist offensichtlich, daß in diesem Fall das eigentliche Versagen, die Versagensart, die spannungsmäßigen Bedingungen, die Werkstoffeigenschaften und die Fehlergröße unbedeutend sind.

Um dies an einem einfachen Beispiel zu verdeutlichen, wird eine Rohrleitung mit $150 \mathrm{~mm}$ Durchmesser und $20 \mathrm{~mm}$ Wanddicke angenommen, die eine schwach korrosive Flüssigkeit unter einem wechselnden Druck bis zu $80 \mathrm{MPa}$ befördern soll. Als Werkstoff wird ein korrosionsbeständiger Stahl mit einer Streckgrenze $R_{p, 0,2}=540 \mathrm{MPa}$ gewählt. Damit ist der korrosive Einfluß vom Tisch.

Die Rohrleitung wird konstruktiv so verankert, daß Eigengewicht und Flüssigkeit vernachlässigt werden können. Die Lebensdauer soll 25 Jahre betragen, was gemäß den betrieblichen Belastungen $5,9 \cdot 10^{3}$ Zyklen mit einer Spannungsamplitude $\Delta \sigma_{1}=300 \mathrm{MPa}, 27 \cdot 10^{3}$ Zyklen mit $\Delta \sigma_{2}=210 \mathrm{MPa}$ und $109 \cdot 10^{3}$ Zyklen mit $\Delta \sigma_{3}=190 \mathrm{MPa}$ entspricht.

Die Lebensdauer der betreffenden Stahlproben unter der zyklischer Belastung von $\Delta \sigma_{1}, \Delta \sigma_{2}$ und $\Delta \sigma_{3}$ soll jeweils $0,031 \cdot 10^{6}, 0,33 \cdot 0^{6}$ und $2,2 \cdot 10^{6}$ Zyklen 